ISAHP 1999, Kobe, Japan, August 12-14, 1999

\title{
COMBINING OF AHP WITH GOAL PROGRAMMING IN DECISION MAKING
}

\author{
Ying Pan \\ Siyang Science and Technology Center \\ 14-2, No.16 Yangfangdian Road, Beijing 100038, China \\ panying@126.com \\ Xuehong Yan \\ Biology Dep. ,Graduate School, Chinese Academy of Sciences \\ No.19 Yuquan Road, Beijing 100039, China \\ biodep@cc5.gsbustc.ac.cn
}

\begin{abstract}
An organization gets a fixed yearly fund in the future five years. The fund will be used to purchase equipment for 20 teams. A five-year plan of procurement and allocation is needed with the purpose that the demand for equipment from all teams be met as much as possible. The paper is about how to make the plan. Goal Programming (GP) is used combining Analytic Hierarchy Process (AHP) that is used to confirm the priorities of variables in GP model..
\end{abstract}

\section{Introduction}

An organization gets a fixed yearly fund in the future five years. The fund will be used to purchase equipment for 20 teams. A five-year plan of procurement and allocation is needed with the purpose that the demand for equipment of all teams be met as much as possible.

The fund dose not meet all needs from the teams, so it should be carefully designed that how much equipment should be equipped to each team.

The paper presents the general steps to make the plan.

\section{General Principle}

Some important teams should be equipped first.

Some types of equipment should be considered as favorite.

If it is possible, needs from any teams should be met.

\section{Working Steps}

\section{General Steps}

The plan is shaped in four working steps as shown in figure 1. 


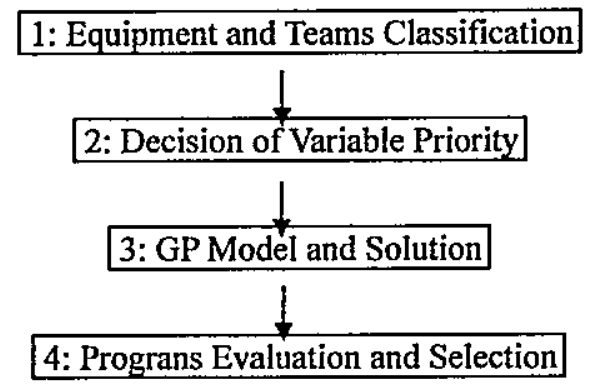

Fig. 1 Working Steps

\section{Step 1: Equipment and Teams Classification}

In order to reduce the number of variables in the GP model, the equipment is classified into several grades according to their prices and utilization ratios by means of Cluster Analysis, and the teams are classified into three levels according to their missions by AHP. After step 1, the number of variables in the GP model is reduced to less than 100 .

\section{Step 2: Decision of Variable Priority}

As the GP method required, each important variable should be appointed a priority in the GP model, so GP could come up with the optimal program. Any changes in the priority of variables may cause some changes in the outcome. Although some variables can be easily assigned priorities, such as high grade equipment of high level teams or low grade equipment of low level teams, most variables are difficult to set. Hence AHP approach, which has been proved to be highly effective in solving similar problems, is employed in this step. After AHP method is used in priority decision, the GP model generates more reliable and flexible programs.

\section{Step 3: GP Model and Solution}

(GP model is omitted)

There are three programs produced by the GP model. They are based on following tentative ideas:

A. Teams are equipped in the level order. The top priority is given to those teams with higher levels. The first lever teams are equipped first.

B. According to equipment grade, a percentage of the amount of equipment asked by ail teams is met first. Then the rest of equipment is considered to those teams in their level order.

C. No matter what level teams belong to, equipment asked by all teams is considered only according to equipment grade. Equipment with the highest grade is taken into account first.

\section{Step 4: Programs Evaluation and Selection}

Three programs are generated in step 3, which are evaluated and selected by using AHP approach just like many successful instances of evaluation and selection that other people have carried out.

\section{References (Omitted)}

